\title{
Advanced EUV negative tone resist and underlayer approaches exhibiting sub-20nm half-pitch resolution
}

\author{
Thomas Gädda*a, Nguyen Dang Luong*a, Markus Laukkanen ${ }^{\mathrm{a}}$, Kimmo Karaste a, Oskari \\ Kähkönen ${ }^{\mathrm{a}}$, Emilia Kauppi ${ }^{\mathrm{a}}$, Dimitrios Kazazis ${ }^{\mathrm{b}}$, and Yasin Ekinci ${ }^{\mathrm{b}}$, Juha Rantala*a \\ aPiBond Oy, Kutojantie 2, 02630 Espoo, Finland \\ ${ }^{\mathrm{b}}$ Paul Scherrer Institute, 5232 Villigen PSI, Switzerland
}

\begin{abstract}
The RLS trade-off of EUV resists has been a major technical issue for high-volume manufacturing using EUVL. Significant attempts to develop of chemically-amplified resists, metal-containing resists, and a variety of other material classes have been made to obtain low LER at high resolution (R) and at a reasonable sensitivity (S). Previously, we have developed and reported work on silanol-containing polyhydrogensilsesquioxane resins and their use as negative tone resists. The developed silanol-containing polymer resists have demonstrated enhanced EUV sensitivity compared to traditional hydrogen silsesquioxane resins, and at the same time maintaining excellent etch properties. The resist may enable a bilayer stack technology in EUVL. Herein we report novel functionalized polyhydrogensilsesquioxane polymers and their use as negative tone resists. These materials exhibit improved LER/LWR and reasonably good EUV sensitivity. In best cases, data suggests no residues or bridging in the non-exposed areas. The optimized resist exhibits sub-20nm halfpitch resolution, low LER (2-3nm), and reasonable sensitivity $\left(82.5 \mathrm{~mJ} / \mathrm{cm}^{2}\right)$. In addition, we also investigated the effect of three organic underlayers for EUV patterning and compared with the silicon substrate.
\end{abstract}

Keywords: structure-property correlation, silicon compounds, EUV lithography, E-beam lithography, RLS trade-off, Non-CAR

\section{INTRODUCTION}

Moore's law has been the paradigm of semiconductor industry for several decades. It indicates that the number of transistors in integrated circuits should double about every two years. This has been achieved by improving material properties and shortening the wavelength of the light used in the exposure step during lithographic patterning. Following this trend, extreme ultraviolet lithography (EUVL) has been considered the most promising method with the aim of achieving sub-20nm half-pitch (HP) resolution using a 13.5nm wavelength of emitted light. ${ }^{1-3}$

The light source and its operation for high volume EUVL has been successfully solved by reaching the target of $250 \mathrm{~W}$ and $>85 \%$ source availability. ${ }^{3}$ One of the main remaining challenges for successful EUVL adaptation and improvement is development resists that can solve the well-known RLS trade-off between resist resolution (R), line-edge roughness (LER), and sensitivity (S). ${ }^{4}$ To enable the implementation of EUV lithography for high-volume manufacturing, EUVL requires high-performance photoresists with high sensitivity $\left(<50 \mathrm{~mJ} / \mathrm{cm}^{2}\right)$ and low line width roughness (LWR) of below $20 \% .^{5}$

Three main resists types that have received most attention in recent years are chemically amplified resists (CAR), metalcontaining resists, and molecular resists. ${ }^{1,6}$ CAR resists have demonstrated good sensitivity but their low resolution and high LWR may be considered drawbacks. The limitations with respect to LWR are mainly due to the stochastic effect of photoacids and photon shot noise. ${ }^{7}$ Potential other drawbacks of CAR may be pattern collapse and a low etch selectivity.

Advances in Patterning Materials and Processes XXXVI, edited by Roel Gronheid,

Daniel P. Sanders, Proc. of SPIE Vol. 10960, 109600B - (C) 2019 SPIE

CCC code: $0277-786 \mathrm{X} / 19 / \$ 18 \cdot$ doi: $10.1117 / 12.2515600$

Proc. of SPIE Vol. 10960 109600B-1 
Metal-containing resist have received significant attention in recent studies. ${ }^{5}$ They have shown several advantages, such as high EUV sensitivity and due to their inorganic content a possibility for stack simplification. The high EUV sensitivity is mostly due to a higher atomic absorption at EUV wavelength compared to other materials. ${ }^{8}$ While metal-containing resists have several advantages, some concerns may arise from impurities and metallic contamination of critical layers. ${ }^{3}$

Hydrogensilsesquioxane (HSQ) has been utilized widely as negative electron-beam resist. However, it possesses low sensitivity $\left(>600 \mathrm{~mJ} / \mathrm{cm}^{2}\right)$ and may require development with highly concentrated developers such as $25 \%$ tetramethylammonium hydroxide (TMAH). Besides, HSQ is known to be relatively unstable during storage and processing, and thus limiting its industrial uses. ${ }^{9}$

In our previous work, we reported silanol-containing hydrogen silsesquioxane-based polymers and used these as EUV negative tone resists. ${ }^{6}$ The silanol-containing resists exhibited low LER of $\sim 2 \mathrm{~nm}$, reasonable sensitivity, and good etch properties. In this paper, we study the behavior of functionalized polyhydrogensilsesquioxane polymers that have different functional groups incorporated in the resin microstructure. These functional groups play a significant role in facilitating the solubility switch by improving reactions in the exposed areas or alternatively facilitate the removal of non-exposed areas during development. The incorporation of different functionalities into the microstructure resulted in sensitivity improvement or LER/LWR and resolution enhancement that have been confirmed by EUVL and e-beam.

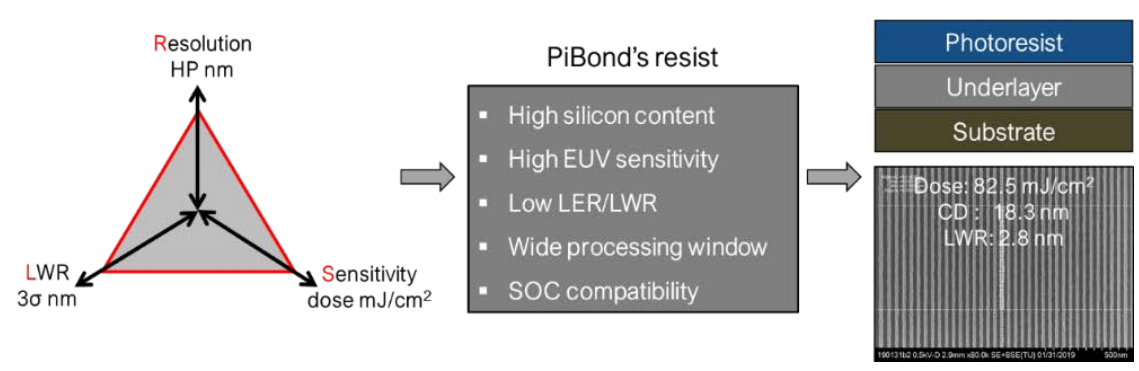

Figure 1. Resist challenge in EUVL and strategies to solve issues at PiBond.

\section{METHODOLOGY}

\subsection{Preparation of Materials}

The materials discussed in this report are described in Table 1. Different functional groups (A, B, C) were incorporated into the reference polymer (PRE 102) structure via copolymerization. In this way, PRE 102A, PRE 102B, and PRE 102C, were obtained, respectively. All polymers were prepared via controlled hydrolysis and condensation of organosilane monomers in suitable solvents, followed by purification steps using a rotary evaporator. All monomers and solvents were distilled prior to use. The obtained materials were then formulated by dilution using PGMEA to achieve desired film thickness. The formulations were finally filtered with a $0.2 \mu \mathrm{m}$ PTFE filter prior to spin coating.

In addition, the effect of three organic underlayers were evaluated to determine the underlayer effect to the polyhydrogensilsesquioxane patterning. 
Table 1. Summary of negative tone resists and underlayers used.

\begin{tabular}{|l|l|c|c|}
\hline \multicolumn{1}{|c|}{ Materials } & \multicolumn{1}{|c|}{ Type } & $\begin{array}{c}\text { Molecular weight } \\
\text { by GPC [g/mol] }\end{array}$ & $\begin{array}{c}\text { Film thickness } \\
\text { [nm] }\end{array}$ \\
\hline PRE 102 reference & Organohydrogensilsesquioxane & $\sim 4000$ & $20 \pm 2$ \\
\hline PRE 102A negative tone resist & Organohydrogensilsesquioxane & $\sim 4000$ & $20 \pm 2$ \\
\hline PRE 102B negative tone resist & Organohydrogensilsesquioxane & $\sim 4000$ & $20 \pm 2$ \\
\hline PRE 102C negative tone resist & Organohydrogensilsesquioxane & $\sim 4000$ & $20 \pm 2$ \\
\hline Underlayers 1, 2,3 & Spin-on-carbon (SOC) & NA & $300 \pm 5$ \\
\hline
\end{tabular}

The structure of synthesized polymers is shown in Figure 2. Functional groups A, B, and C were incorporated in the polymer microstructure through controlled hydrolysis and condensation reactions. In all cases, $10 \mathrm{~mol}-\%$ or less of the functional precursor was used. Functional groups A and B are used to enhance resin reactivity under EUV exposure. Group $\mathrm{C}$ enhances polymer solubility in the developer.

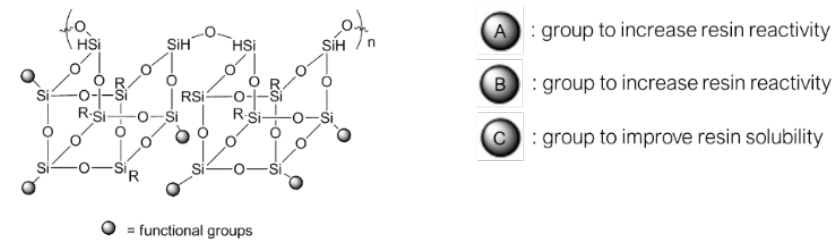

Figure 2. structure of chemically functionalized HSQ resins with four different functionalities which are A, B, and C.

\subsection{Film Characterization and Patterning}

EUV lithography was carried out using a XIL-II EUV tool at Paul Scherrer Institute, Villigen, Switzerland. Process steps used in the in EUV lithography evaluation are summarized in Table 2. All resists materials tested exhibited a post coating delay for at least 24h. During 24h of storage the coatings perform similarly. In addition, the aging study has been carried out to evaluate the stability of resist solutions. The molecular weight data obtained by GPC analysis confirms the stability of developed resists at $-18{ }^{\circ} \mathrm{C}$ and $4{ }^{\circ} \mathrm{C}$ for at least two months and at $23^{\circ} \mathrm{C}$ for at least seven days.

Scanning electron micrographs were obtained via Zeiss Supra VP55 high resolution field emission scanning electron microscope for e-beam samples, and Hitachi CD SEM was used for EUV samples.

E-beam lithography was carried out on a Vistec EPBG5000pES tool. The e-beam doses were varied from 100 to 1200 $\mu \mathrm{C} / \mathrm{cm}^{2}$ at $100 \mathrm{kV}$ and current of $0.5-1 \mathrm{nA}$. The development steps were carried out similarly as described above for the EUV lithography.

For patterning of PRE 102 reference resist on an organic underlayer: SOC was spin coated on silicon wafer at $1500 \mathrm{rpm}$ for 20 seconds. The obtained coating was baked at $150{ }^{\circ} \mathrm{C}$ for 1 min and $400{ }^{\circ} \mathrm{C}$ for 2 minutes.

Table 2. Process steps in lithography experiments.

\begin{tabular}{|l|l|l|}
\hline Process & Parameters & Details \\
\hline Spin coat & $1500 \mathrm{rpm}, 20$ seconds & $2 \mathrm{~mL}$ dispense on 4 -inch wafer \\
\hline Pre Bake & $80-120 \mathrm{C}, 60$ seconds & Hot plate, contact \\
\hline EUV exposure & $2-100 \mathrm{~mJ} / \mathrm{cm} 2$ & Dose to size at PSI \\
\hline Development & TMAH $2.38 \%, 1-2 \mathrm{~min}$ & Immersion \\
\hline Rinse & Deionized water, $10 \times 3$ times & Manual spray \\
\hline Dry & $\mathrm{N}_{2} /$ compressed air & Manual \\
\hline
\end{tabular}




\section{RESULTS AND DISCUSSION}

\subsection{EUV sensitivity improvement with PRE 102A resist}

The addition of functional group A into the polyhydrogensilsesquioxane (PRE 102A) microstructure significantly improved the sensitivity of the resist material as shown in Figure 3. The effect was observed both in EUVL and e-beam tests when compared to a reference which did not contain the reactive group. As demonstrated herein, incorporating 10 mol-\% or less of functional group A into the polymer has increased sensitivity significantly (89\%) in EUV exposure. Similar effects were seen when material was patterned using e-beam direct exposure - i.e. the dose needed for patterning of the resist decreased significantly (Figure 3). The decrease in required dose to pattern the material, effectively means that the functional group provides an additional pathway for crosslinking and thus a lower dose is required.

On the other hand, a reactive group, such as group A, may enhance the resist reactivity too much. Based on our observations, this is in fact observed in EUV exposures. The residue in non-exposed areas were significant in EUVL even with very low EUV doses $\left(7.2 \mathrm{~mJ} / \mathrm{cm}^{2}\right)$. The same was not seen for the e-beam exposures which suggests that the targeted line width also has an impact on the patterning result. In EUVL experiments the targeted half pitch was 22nm, while in ebeam tests it was $50 \mathrm{~nm}$. An increase in the concentration of functional group A in the microstructure lead to similar challenges where the non-exposed areas contained significant amount of unresolved polymer.

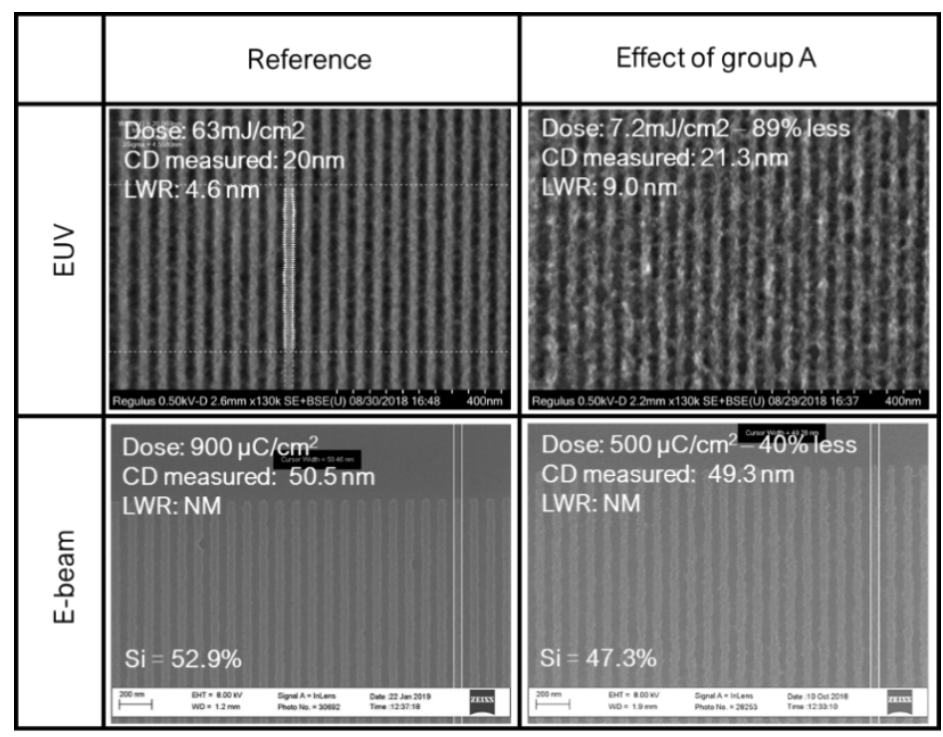

Figure 3. EUV performance of PRE 102A with functional group A, demonstrating significantly improved sensitivity compared with the reference sample, which did not contain the functional group.

\subsection{LER/LWR improvement with PRE 102B resist}

Both e-beam and EUV lithography was carried out for PRE 102B to understand the effect of the functional group B in a polyhydrogensilsesquioxane (figure 4). We expected functional group B to behave in a similar way as functional group A. Based on initial e-beam tests, it appeared that a dose decrease could be obtained when patterning 50nm L/S patterns with e-beam. However, the dose decrease $\left(100 \mu \mathrm{C} / \mathrm{cm}^{2}\right)$ with functional group B was not equally substantial as seen with functional group A $\left(400 \mu \mathrm{C} / \mathrm{cm}^{2}\right)$. The amount of functional group B in experiments was same as for group A, $10 \mathrm{~mol}-\%$ or less.

The dose required for patterning using a polyhydrogensilsesquioxane containing a functional group B in EUV exposures was slightly higher $\left(82.5 \mathrm{~mJ} / \mathrm{cm}^{2}\right)$ than for the reference material $\left(77.5 \mathrm{~mJ} / \mathrm{cm}^{2}\right)$. Also, the material is slightly more 
underexposed compared to the reference material when patterning 22nm half pitch $\mathrm{L} / \mathrm{S}$ structures. Thus, the dose decrease seen in e-beam experiments could not be duplicated in EUVL. The reason for this is not fully understood. On the other hand, we found to our surprise that the use of functional group B improved the contrast of the polyhydrogensilsesquioxane resist. This is seen as a clear decrease in residues and bridging of the non-exposed areas. The improved contrast also

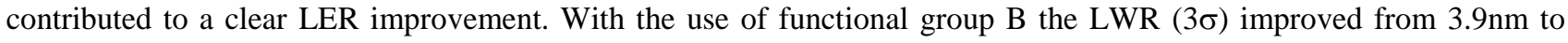
$2.8 \mathrm{~nm}$.

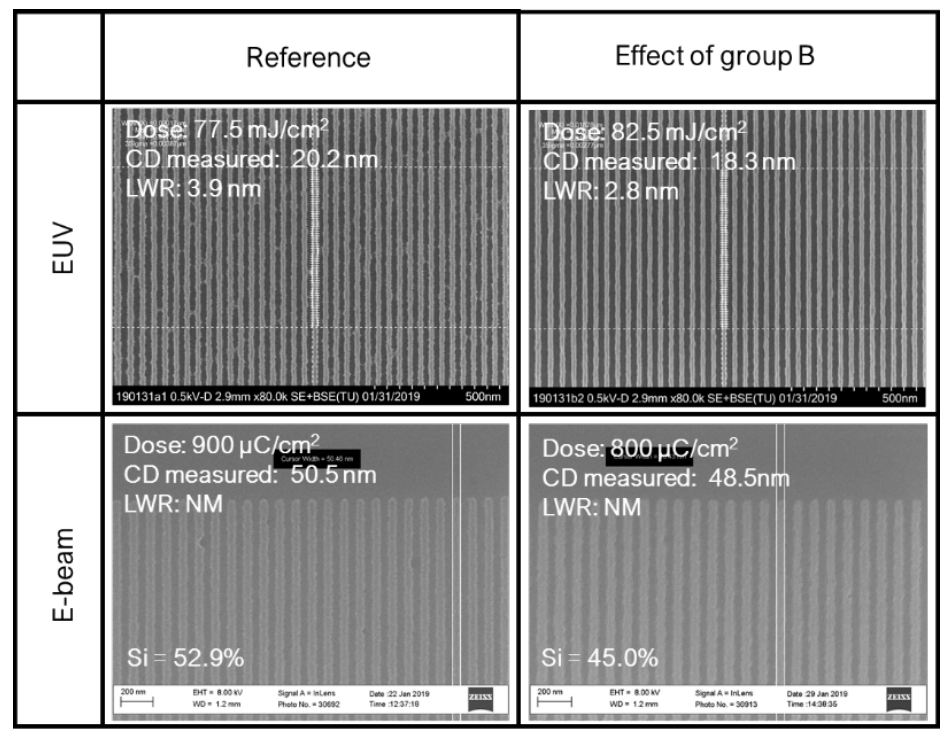

Figure 4. EUV performance of PRE 102B with 5\% and 10\% in mole of functional B, demonstrating significantly improved LER and overcoming the bridging/scumming issue as seen clearly in the PRE 102 reference.

\subsection{LER/LWR improvement with PRE 102C resist}

Functional group $\mathrm{C}$ used in the resin microstructure is different compared to A and B. Functional group C improves the resist solubility to the developer and hence could improve the contrast of the resist pattern. In fact, this was observed and incorporation a functional $\mathrm{C}$ in the polymer structure resulted in an improvement in LER/LWR. The improvement was similar as seen for functional group B, i.e. the LWR decreased to 2.8nm (Figure 5). While LWR improved the dose needed to achieve patterns increased. At a dose of $82.5 \mathrm{~mJ} / \mathrm{cm}^{2}$ the $22 \mathrm{~nm}$ half pitch lines were still under exposed.

\begin{tabular}{|c|c|c|}
\hline & Reference & Effect of group C \\
\hline 己 & 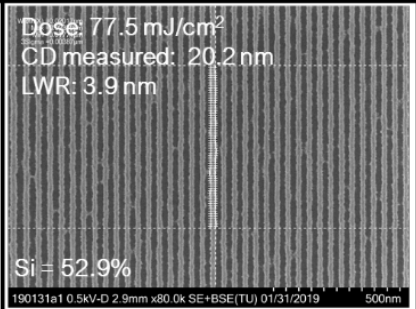 & 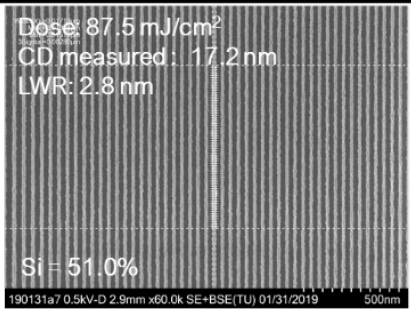 \\
\hline
\end{tabular}

Figure 5. EUV performance of PRE 102D with 1\% and 2\% in mole of functional D, demonstrating improved LER and overcoming the bridging/scumming issue as seen clearly in the PRE 102 reference. 


\subsection{Processing windows comparisons}

In patterning tests, we found out that the reference material exhibited a process window which was relatively narrow. The reference material showed reasonable results (LWR, dose) when underexposed. However, when doses used to meet printed lines that matched the mask dimensions, significant residues and bridging of lines appeared. Thus, an improvement in the process window would be of significant interest. Exposure trials for resists with functional groups B and C appear to also improve the process window. Polyhydrogensilsesquioxanes containing functional groups B or C did not exhibit residues or bridging at optimal dose or slight overexposure.

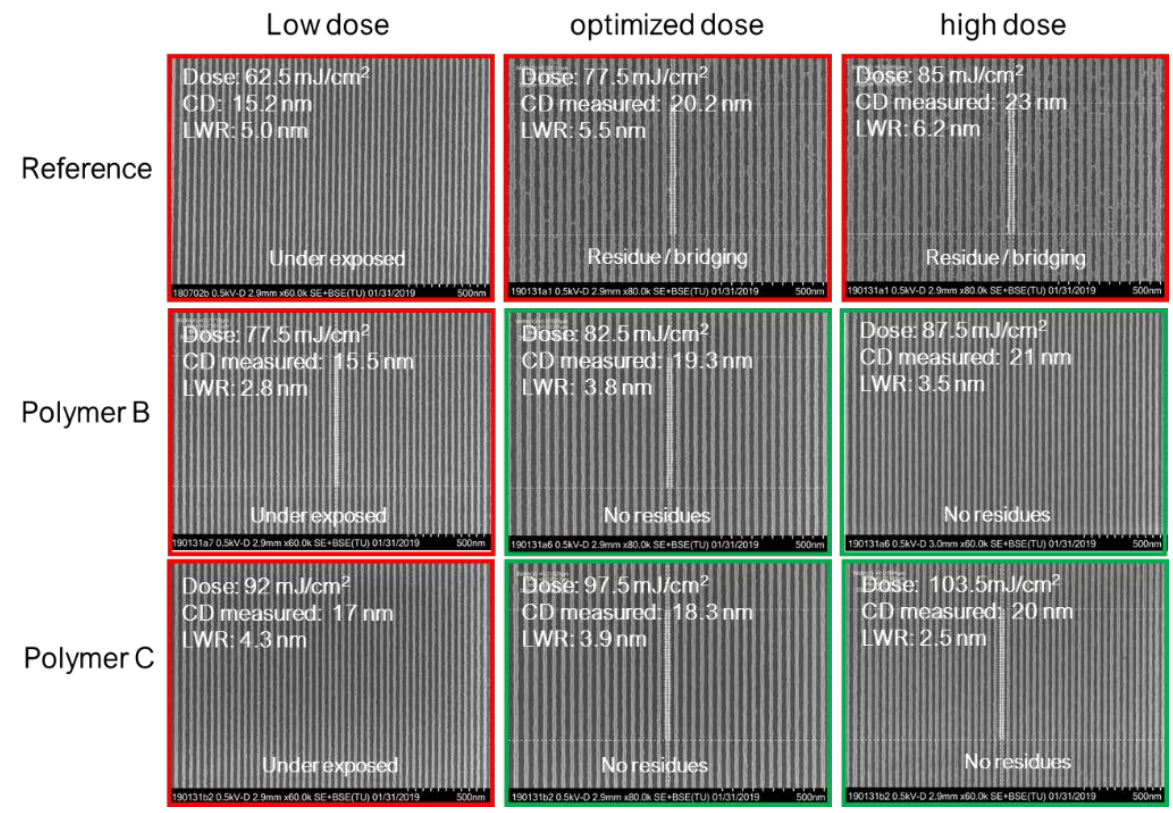

Figure 6. Processing windows comparing EUV patterning performance of PRE 102D, PRE 102B, and PRE 102 reference, demonstrating wider processing windows for PRE 102B and PRE 102D.

\subsection{Underlayer effect on EUV patterning performances}

Underlayer used may have significant effect on the patterning outcome of a polyhydrogensilsesquioxane, and was a subject of a brief investigation. We studied the effect of three organic underlayers on the performance of the PRE 102 reference in EUVL, and compared with result on silicon substrate. A 22-nm line pattern was used in all cases. SEM images and calculated data (Figure 7) show that the underlayer may have a profound effect on the patterning result. Results using underlayer 1 and 3 suggests that with a proper choice of underlayer, one can actually improve the results. Exposure trials on underlayer 1 and 3 show less residues and bridging of lines compared to the silicon reference. A slightly higher dose was needed when the exposure was carried on an organic underlayer compared to silicon. On the other hand, exposure on underlayer 2 yielded worse results. This suggests that the functional groups in the underlayer will have a significant effect to the patterning process and also adhesion of the resist to the substrate. The effect of each functional group present in the underlayer is not fully understood and is presently being investigated further. 

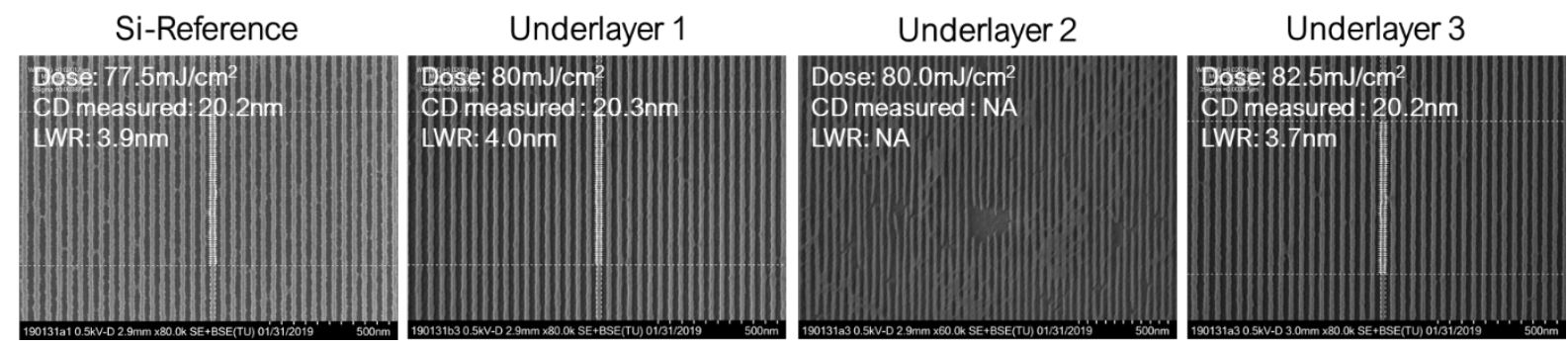

Figure 7. Underlay effect on EUV patterning performance wherein silicon is used as reference.

\section{SUMMARY AND CONCLUSIONS}

We have reported herein novel EUV resists based on functional polyhydrogensilsesquioxanes. Controlled hydrolysis and condensation reactions permit synthesis if materials with improved properties. The developed materials work as negative tone resists based on the polymerization that results in solubility switch of the materials rendering the exposed areas insoluble to the developer. The resists exhibit low LWR $(-2-3 \mathrm{~nm})$, resonable sensitivity $\left(40-90 \mathrm{~mJ} / \mathrm{cm}^{2}\right)$, and ability to form pattern by industry standard aq. TMAH development processes after exposure. Especially, the functional polyhydrogensilsesquioxanes showed improved performance by eliminating residues and bridges in the non-exposed areas. The materials further exhibit high silicon content which may permit the use of a bilayer stack to simplify the lithographic process.

\section{ACKNOWLEDGMENTS}

This project has received partial funding from the EU-H2020 research and innovation programme under grant agreement No 654360 having benefitted from the access provided by the Paul Scherrer Institut in Villigen within the framework of the NFFA-Europe Transnational Access Activity.

\section{REFERENCES}

[1] Bakshi V., EUV lithography, Bellingham, Washington, USA: SPIE Press, (2018).

[2] Mojarad, N., Gobrecht, J. and Ekinci, Y., "Beyond EUV lithography: a comparative study of efficient photoresists' performance,” Sci. Rep. 5, 9235 (2015).

[3] De Simone, D., Vesters, Y. and Vandenberghe, G., "Photoresists in extreme ultraviolet lithography (EUVL)," Adv. Opt. Technol. 6, 163-172 (2017).

[4] Higgins, C. D., Szmanda C. R., Antohe, A., Denbeaux, G., Georger, J. and Brainard R. L. "Resolution, line-edge roughness, sensitivity tradeoff, and quantum yield of high photo acid generator resists for extreme ultraviolet lithography,” Jpn. J. Appl. Phys. 50, 1-8 (2011).

[5] Wu L., Baljozovic M., Portale G., Kazazis D., Vokenhuber M., Jung T., Ekinci Y., and Castellanos S., "Mechanistic insights in Zr- and Hf-based molecular hybrid EUV photoresist,” J. Micro/Nanolith. MEMS MOEMS 18(1), 013504 (2019).

[6] Rantala J., Gädda T., Laukkanen M., Luong N.D., Karaste K., Kazazis D., Ekinci Y., “New resist and underlayer approaches toward EUV lithography,” Proc. SPIE 10809, International Conference on Extreme Ultraviolet Lithography 2018, 108090X (2018).

[7] Naulleau P., Anderson C., and George S., "EUV resists: Illuminating the challenges,”Journal of Photopolymer and Technology. 24, 637-642 (2011).

[8] Jiang, J., Zhang, B., Yu, M., Li, L., Neisser, M., Chun, J.S., Giannelis, E.P. and Ober, C.K., “Oxide Nanoparticle EUV (ONE) Photoresists: Current Understanding of the Unusual Patterning Mechanism,” J. Photopolym. Sci. Technol., 28(4), 515-518 (2015).

[9] Yang, J. K. W., Cord, B., Duan, H. and Berggren. "Understanding of hydrogen silsesquioxane electron resist for sub-5-nm-half-pitch lithography,” J. Vac. Sci. Technol. B Microelectron. Nanom. Struct. 27, 2622 (2009). 